\title{
Neurological paraneoplastic syndromes in patients with small cell lung cancer. A prospective survey of 150 patients
}

\author{
G M Elrington, N M F Murray, S G Spiro, J Newsom-Davis
}

\begin{abstract}
One hundred and fifty patients presenting with small cell lung cancer (SCLC) to chest physicians, were assessed neurologically. Neuromuscular or autonomic deficits were common and occurred in up to $44 \%$ of cases. Weakness, dry mouth, and weight loss were not mutually independent and may represent the syndrome formerly described as carcinomatous neuromyopathy. By contrast, undoubted paraneoplastic syndromes were much less commonly detected. Two patients had the Lambert-Eaton myasthenic syndrome (LEMS) and one had subacute sensory neuropathy (SSN). In these patients, neurological symptoms antedated other manifestations of cancer, by between six and 17 months. The $95 \%$ confidence interval for the prevalence of LEMS or SSN among SCLC patients was $0-4 \%$, consistent with the results of previous retrospective or smaller studies: summing these, the overall prevalence of LEMS among SCLC patients is close to $3 \%$, which implies about 250 new cases per annum in England and Wales. If LEMS and SSN are the least uncommon neurological paraneoplastic syndromes in SCLC patients, this may reflect the accessibility of motor nerve terminals and dorsal root ganglia to cross-reactive anti-tumour cell antibodies.
\end{abstract}

Small cell lung cancer (SCLC) is the tumour most commonly associated with a range of neurological paraneoplastic syndromes; $25 \%$ of all patients with lung cancer have this cell type. ${ }^{1}$ Henson and Urich ${ }^{2}$ defined syndromes as paraneoplastic if associated with cancer and not due to the presence of cancer in the affected organ. Other tumours are associated with individual paraneoplastic syndromes, such as malignancies of the female genital tract with cerebellar degeneration, ${ }^{3}$ neuroblastoma with opsoclonus, ${ }^{2}$ and Hodgkin's disease with demyelinating neuropathy. ${ }^{4}$ Such specific associations are also seen with SCLC, but the range of neurological syndromes complicating this common and highly malignant cancer include the Lambert-Eaton myasthenic syndrome (LEMS), ${ }^{5}$ subacute sensory neuropathy (SSN), ${ }^{6}$ myelopathy, ${ }^{7}$ cerebellar degeneration, ${ }^{8}$ and encephalopathy. ${ }^{6}$ These syndromes may coexist. ${ }^{2}$ Opsoclonus, ${ }^{9}$ and retinal degeneration ${ }^{10}$ are also reported, less commonly, in SCLC patients.

The immunopathology of LEMS has recently been reviewed. ${ }^{11}$ LEMS appears to be caused by the downregulation of presynaptic voltage gated calcium channels (VGCCs), following cross-linking by divalent antiVGCC IgG antibodies. VGCC are also present in the cell membrane of SCLC, which, in immunogenetically susceptible individuals, may trigger the formation of anti-VGCC antibodies.

The frequency at which paraneoplastic neurological disorders occur in association with SCLC is uncertain, and most data have been obtained in retrospective studies. These syndromes might easily be overlooked, particularly in the case of LEMS, ${ }^{5}$ in patients known to have a malignant neoplasm. We therefore undertook a prospective survey to assess the prevalence of neurological paraneoplastic syndromes among patients with SCLC.

\section{Methods}

One hundred and fifty consecutive patients with a tissue diagnosis of SCLC were assessed clinically at five hospitals participating in trials of chemotherapy for SCLC. Mean (SD) age was $64(7 \cdot 4)$ years and $79 \%$ were male. Thirty five per cent had limited disease, that is, confined to one hemithorax, or with bilateral hilar involvement. Eighty per cent were recruited to trials of chemotherapy, but all patients were assessed before the administration of cytotoxic drugs. Tissue diagnosis was achieved following fibreoptic bronchoscopy in 122 cases, of which 114 had a positive biopsy; the other eight were diagnosed on cytological examination of bronchial washings. Nine cases were diagnosed by sputum cytology alone, and eleven from other non-bronchoscopic biopsies. All patients had been given a diagnosis of SCLC, but four also had co-existent nonSCLC bronchogenic tumours.

A standard assessment protocol was used. Background information about the presentation of the tumour, and its diagnosis, was established from clinical case notes. Enquiry about past medical history and family history included direct questions about diabetes, thyroid disease, and neurological disease. Current and recent medication was recorded. Direct questions were asked to establish the presence or absence of weakness, anorexia, weight loss, dry mouth, sphincter difficulty, erectile impotence, diplopia, and ptosis. A 
detailed neurological assessment was performed, but in 20 cases this was limited by the patient's inability to stand. Tendon reflexes were elicited before assessment of muscle power, and, in subjects with diminished reflexes, again after 15 seconds sustained muscular contraction, in at least two muscles. Cardiovascular reflexes were assessed in a standard manner: the resting, supine blood pressure and heart rate were measured. Patients then stood, and measurements were repeated: standing blood pressure was measured, and heart rate counted, starting 15 to 20 seconds after the patient began to stand.

Patients had electrophysiological testing if a diagnosis of LEMS or another neurological paraneoplastic syndrome was suspected from the clinical assessment $(n=7)$. To establish a diagnosis of LEMS, the amplitude of the compound muscle action potential (CMAP) was measured in abductor digiti minimi in response to supramaximal stimulation of the ulnar nerve, before and after 15 seconds maximal voluntary contraction. In some cases, the response to trains of stimuli at 5 and $20 \mathrm{~Hz}$ was examined, and single fibre electromyography was performed.

Electrophysiological criteria for the diagnosis of LEMS were taken from the data of Newson-Davis and Murray: $:^{12}$ resting CMAP amplitude $<8.4 \mathrm{mV}$, and increment $>25 \%$ following 15 seconds maximal voluntary contraction (MVC) being abnormal (\% increment $=100 \times$ [amplitude post MVC - initial amplitude] + initial amplitude). These criteria were stringent and would, for example, have excluded two of fifty subjects who were accepted as having LEMS on other grounds, such as a characteristic clinical picture and abnormal single fibre electromyography. ${ }^{5}$

All clinical data were tested for lack of mutual independence, using Chi squared with Yates' correction. For simplicity of expression, features found not to be mutually independent will be referred to as associated. Because multiple comparisons were made, the threshold of significance was set high at $\mathrm{p}<0.001$.

\section{Results}

The frequency of neuromuscular symptoms and signs among the 150 SCLC patients is shown, as a percentage, in table 1 . Anorexia and weight loss were the commonest nonrespiratory symptoms, followed by dry mouth, and erectile impotence among male subjects (sexual dysfunction was not sought among the female subjects). Physical examination revealed weakness in some subjects who had not complained of this symptom, and overall, $44 \%$ had either subjective or objective weakness, or both.

Some symptoms were mutually associated, especially anorexia and weight loss $(\mathrm{p}<0.00001)$, supporting the accuracy of patients' histories. Weight loss was also associated with dry mouth $(\mathrm{p}<0.001)$. All three measures of weakness (symptomatic,
Table 1 Neuromuscular and somatic clinical features of 150 SCLC patients

\section{$\%$ of total}

Symptoms:
Anorexia
Weight loss
Erectile impotence (males)
Dry mouth
Weakness
Sphincter disturbance
Sweating change
Visual change
Physical signs:
Unable to rise from squatting position
Sensory change
Brisk reflexes
Absent reflexes
Diminished reflexes
Weakness
Ataxia or nystagmus
Post tetanic potentiation

5

53
51
44
41
31
24
21
6

'One or more measures of weakness was present in $44 \%$ of subjects.

${ }^{2}$ Excluding inability to rise from squatting position.

objective proximal weakness, and inability to rise from a squatting position) were associated with each other $(p<0.001)$; symptomatic and proximal weakness were each associated with dry mouth $(\mathrm{p}<0.001)$. These data are shown in the figure.

Other clinical parameters were mutually independent. Abnormal cardiovascular reflexes were common: $11 \%$ had a postural systolic blood pressure fall of $>29 \mathrm{mmHg}$, with a borderline fall of $11-29 \mathrm{mmHg}$ in a further $26 \% .^{13}$ In $18 \%$ of subjects, the pulse rate failed to rise on standing.

LEMS was suspected from the clinical assessment in seven patients. Their details are shown in table 2. Six of these agreed to have EMG studies, and diagnostic features of LEMS were present in two; these two were also distinguished by their long histories of weakness, 11 and 17 months before the diagnosis of SCLC.

One patient had SSN. This 63 year old woman presented with a six month history of painful sensory loss in all four limbs, and also described dry mouth and weakness; examination revealed distal sensory loss to pain, and joint position sense impairment with sensory ataxia. Sensory action potentials were absent from right median, ulnar, radial, and sural nerves; motor conduction velocities were normal. Right ulnar CMAP was slightly reduced, at $7.0 \mathrm{mV}$, but there was no pathological increment.

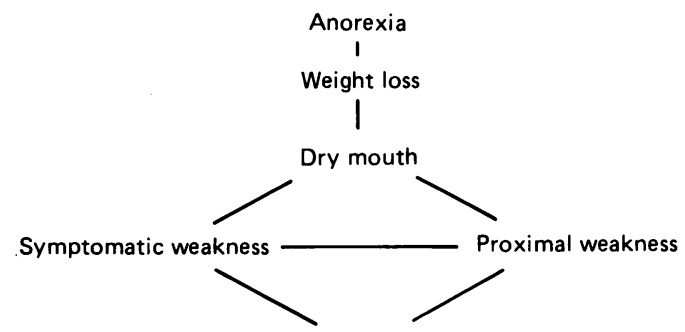

Unable to rise from squatting position

Figure Clinical features, for which the null hypothesis that they were mutually independent may be rejected ( $p<0.001$; Chi squared, with Yates' correction), are shown linked. 
Table 2 SCLC patients in whom a diagnosis of LEMS was suspected on clinical grounds

\begin{tabular}{|c|c|c|c|c|c|c|c|c|c|}
\hline Sex & $\begin{array}{l}\text { Length of } \\
\text { symptoms (months) }\end{array}$ & $\begin{array}{l}\text { First } \\
\text { symptom }\end{array}$ & Weakness ${ }^{t}$ & $\begin{array}{l}\text { Dry } \\
\text { Mouth' }\end{array}$ & $\begin{array}{l}\text { Erectile impotence } \\
\text { (males) }\end{array}$ & $\begin{array}{l}\text { Tendon } \\
\text { reflexes }^{3}\end{array}$ & $\begin{array}{l}\text { Clinical } \\
P T P^{4}\end{array}$ & $\begin{array}{l}C M A P \\
(m V)^{5}\end{array}$ & $\begin{array}{l}\text { Increment } \\
\%\end{array}$ \\
\hline $\begin{array}{l}\mathbf{F} \\
\mathbf{M} \\
\mathrm{F} \\
\mathrm{M} \\
\mathrm{M} \\
\mathrm{F} \\
\mathrm{M}\end{array}$ & $\begin{array}{r}17 \\
11 \\
4 \\
3 \\
2 \\
2 \\
3\end{array}$ & $\begin{array}{l}\text { Weakness } \\
\text { Weakness } \\
\text { Dry mouth } \\
\text { Weakness } \\
\text { Weakness } \\
\text { Haemoptysis } \\
\text { Dyspnoea }\end{array}$ & $\begin{array}{l}++ \\
++ \\
++ \\
++ \\
++ \\
+ \\
+\end{array}$ & $\begin{array}{l}++ \\
+ \\
+++ \\
++ \\
+ \\
+++ \\
++\end{array}$ & $\begin{array}{l}+ \\
+ \\
+ \\
-\end{array}$ & $\begin{array}{l}-\bar{t} \\
\overline{+} \\
+ \\
+ \\
+ \\
+\end{array}$ & $\begin{array}{l}++ \\
+ \\
++ \\
+ \\
+ \\
-\end{array}$ & 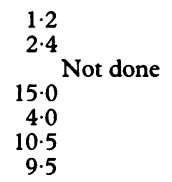 & $\begin{array}{l}610 \\
212 \\
\text { Not done } \\
12 \\
1 \\
0\end{array}$ \\
\hline
\end{tabular}

'Symptoms scored as mild $(+)$, moderate $(++)$, or severe $(+++)$.

${ }^{2}$ Erectile impotence scored as present $(+)$ or absent $(-)$.

${ }^{3}$ Tendon reflexes scored as absent $(-)$, or present without reinforcement $(+)$.

${ }^{4}$ Post tetanic potentiation of muscle power or of tendon reflexes; scored as absent $(-)$, barely present $(+)$, or clearly present $(++)$.

${ }^{5}$ Amplitude of compound muscle action potential (CMAP); normal $>8.4 \mathrm{mV}(12)$.

${ }^{6}$ Increment of CMAP following 15 seconds maximal voluntary contraction; normal $<25 \%(12)$.

Twenty four subjects had sensory impairment, most commonly distal vibration sense loss, and 15 were areflexic. These two features were not significantly associated $(0.01<\mathrm{p}<0.05)$.

Four patients had ataxia of the trunk and limbs, of whom two had CT evidence of cerebellar brain metastases. In one case without radiological evidence of metastases, the ataxia was mild, in the other it was of sudden onset with subsequent improvement, and therefore attributed to stroke. Three patients had nystagmus, without ataxia.

In patients with LEMS and SSN, neurological symptoms antedated other manifestations of SCLC, but in none of these three patients was the correct neurological diagnosis suspected before recruitment to this survey. The $95 \%$ confidence interval for their combined prevalence was $0-4 \%$.

Four patients presented with symptoms from cerebral metastases, demonstrated on CT brain scan. Their otherwise asymptomatic thoracic lesions were demonstrated by chest radiograph and confirmed histologically. Thus the $95 \%$ confidence interval for the prevalence of SCLC presenting with cerebral metastases was $0-5 \%$.

\section{Discussion}

In this prospective study of 150 patients presenting with SCLC to chest physicians, three patients (95\% confidence interval: $0-4 \%$ ) presented with neurological paraneoplastic syndromes (two LEMS, one SSN), similar to the number presenting with brain metastases. No patients were thought to have paraneoplastic cerebellar degeneration (PCD), although it could be argued that mild, non-progressive ataxia or nystagmus could represent PCD; but PCD is typically severe and progressive. Hawley et al ${ }^{14}$ also found no cases of PCD in their survey of 71 SCLC patients.

Autonomic disturbance has been reported as a specific paraneoplastic syndrome, ${ }^{15}{ }^{16}$ but none of our patients presented with cardiovascular autonomic failure although many complained of dry mouth and of erectile impotence. Postural hypotension was perhaps commoner than might have been expected; subgroup analysis on the basis of other causes of postural hypotension (drugs, diabetes, cardiac disease, or hyponatraemia) suggested that conditions other than SCLC did not account for these findings. On average, rise in standing pulse rate was lower ( $p<0.001$ : Student's $t$ test) than in a previously reported group of healthy controls of similar age distribution, ${ }^{17}$ although the sig- nificance of this difference fell to $p<0.01$ when subjects with other sufficient cause of autonomic disturbance were excluded from the analysis.

Dry mouth has been reported as a specific feature of LEMS, useful in the differential diagnosis from myasthenia gravis. ${ }^{5}$ The presence of this symptom in $41 \%$ of SCLC patients limits its value in the diagnosis of weakness in patients known to have SCLC.

In this sample of 150 patients, the finding of weakness in $44 \%$ of the subjects is likely to be representative of all SCLC patients. The lower incidence of LEMS and of SSN is clearly subject to a large error, and the $95 \%$ confidence interval of $0-4 \%$ implies that, on these data, any survey of SCLC patients might yield no cases.

The $95 \%$ confidence interval could be narrowed by studying a larger sample of SCLC patients; but if our data are representative of all cases of SCLC, a sample size of 1000 SCLC patients would be required to reduce the range of the confidence interval to $\pm 1 \%$.

Previous studies have addressed this question, either on a smaller scale or in retrospective series. Lambert's group $^{18}$ estimated that LEMS affected $6 \%$ of SCLC subjects, on the basis of case findings in an estimated total lung cancer population among whom $16 \%$ were thought to have SCLC. This $16 \%$ is an underestimate: a review of 449 old pathological specimens found that SCLC had been diagnosed in $7 \%$ of all lung cancer patients, but $22 \%$ had this cell type according to modern criteria. ${ }^{19}$ Lambert's SCLC population was therefore larger than his estimate, thus reducing the apparent prevalence of LEMS in his series, which was also likely to have been influenced by selection bias. It was acknowledged that the $6 \%$ figure was a rough estimate, and seemed inconsistent with the data of Balz ${ }^{18}$ who studied 29 SCLC patients electrophysiologically and found no cases of LEMS. Similarly, Croft and Wilkinson, ${ }^{20}$ in a prospective series, found no cases of "myasthenic neuromyopathy" among 319 cases of lung cancer, of whom $25 \%$ would have had SCLC.

More recent, prospective studies, have been smaller than our series. An electrophysiological study of 71 SCLC patients detected two cases of LEMS. ${ }^{14}$ A clinical study found two cases among 35 patients with SCLC. ${ }^{20} \mathrm{~A}$ recent retrospective necropsy series of 85 SCLC patients contained five with LEMS; ${ }^{22}$ but LEMS patients are more likely to have a necropsy than non-LEMS SCLC patients.

If the present series is added to previous 
Table 3 Reported prevalence of LEMS among SCLC patients

\begin{tabular}{lccl}
\hline Authors & $\begin{array}{l}\text { LEMS } \\
\text { cases }\end{array}$ & $\begin{array}{l}\text { SCLC } \\
\text { population }\end{array}$ & $\begin{array}{l}\text { LEMS } \\
\text { prevalence (\%) }\end{array}$ \\
\hline${\text { Lambert } \text { et al } 1961^{18}}_{\text {Balz }}^{2}$ & 12 & $328^{1}$ & 4 \\
${\text { Croft and Wilkinson } 1965^{20}}_{\text {Hawley } \text { et al } 1980^{14}}$ & 0 & 29 & 0 \\
De la Monte et al $1984^{22}$ & 0 & $80^{23}$ & 0 \\
Gomm 1986 & 2 & 71 & 3 \\
Present series & 5 & 85 & 6 \\
Totals & 2 & 35 & 6 \\
& 23 & 150 & 2 \\
\hline
\end{tabular}

Original report assumes SCLC as $16 \%$ of estimated lung cancer population; assumption revised here to $25 \%$ - see text for explanation

${ }^{2}$ Reported by Lambert et al, 1961

${ }^{3}$ Assuming SCLC as $25 \%$ of all 319 cases of lung cancer.

data, then 23 cases of LEMS have been detected among 778 SCLC patients, an overall prevalence of $3 \%$. These figures are shown in table 3.

The annual death rate from all types of lung cancer in England and Wales is $35000,{ }^{23}$ of whom $25 \%$ have SCLC. If $3 \%$ of SCLC patients have LEMS, then there will be 262 new cases of SCLC associated LEMS each year. The symptoms of neurological paraneoplastic syndromes typically predate other manifestations of cancer, ${ }^{3}$ as was also observed in this survey; yet although the survey raised local awareness of the existence of these syndromes, the correct neurological diagnosis was not made until after SCLC was detected. It is likely that the majority of neurological paraneoplastic syndromes escape diagnosis in SCLC patients.

SCLC carries a grave prognosis, which nonSCLC LEMS does not. ${ }^{5}$ There is therefore a selection bias towards non-SCLC LEMS in neurological practice. The ratio of paraneoplastic to non-paraneoplastic LEMS is likely to be greater than the $2: 1$ ratio previously reported. ${ }^{5}$

Weakness was the single commonest neurological abnormality among these SCLC patients. Weakness, weight loss, and dry mouth were not mutually independent clinical features, perhaps reflecting a common cause. Familiarity with LEMS might suggest that diagnosis in SCLC patients complaining of weakness and dry mouth; but over one third of SCLC subjects were weak, and less than $2 \%$ had LEMS. These symptoms were associated with weight loss, which is known to correlate with neurological abnormalities. ${ }^{14}$

Before the introduction of the concept of paraneoplastic syndromes, the term "neuromyopathy" was used. This was introduced by Brain and Henson ${ }^{24}$ to describe dysfunction at any level in the nervous system, in cancer patients. Croft and Wilkinson ${ }^{19}$ found evidence of neuromyopathy in $15 \%$ of lung cancer patients. Shy and Silverstein ${ }^{25}$ restricted the term to describe proximal muscle weakness and reduced myotactic reflexes, yet still found it to be the commonest neurological abnormality in cancer patients. The term neuromyopathy is ambiguous implying neither neuropathy nor myopathy, both of which occur in cancer patients, although it has been shown that these changes were more closely related to weight loss than to the presence of cancer. ${ }^{14}$ Our data also suggest that weakness in SCLC patients may be a consequence of weight loss.

LEMS and SSN are both associated with antibodies to SCLC antigens which recognise determinants at motor nerve terminals and at dorsal root ganglia, respectively. Other neurological paraneoplastic syndromes which also associate with crossreactive antibodies were not detected in this survey. The greater frequency of LEMS and SSN among neurological paraneoplastic syndromes may reflect the fact that motor nerve terminals and dorsal root ganglia lack the blood-nerve and blood-brain barriers that may protect other neural antigens. ${ }^{26}$

GME gratefully acknowledges the support of the Cancer Research Campaign, who also funded the trials of chemotherapy, upon which recruitment to this survey was based.

Preliminary results from this study were presented to the Association of British Neurologists, 27-29 October 1988.

1 Ianuzzi MC, Scoggin CH. Small cell lung cancer. Am Rev Resp Dis. 1986;134:593-608.

2 Henson RA, Urich H. Cancer and the nervous system. Oxford: Blackwell Scientific, 1982:311-621.

3 Dropcho EJ. The remote effects of cancer on the nervous system. Neurological clinicas, Vol 7. Philadelphia: WB Saunders, 1989:579-603.

4 Lisak RP, Mitchell M, Zweiman B, Asbury AK. Guillain Barré syndrome and Hodgkin's disease; three cases with immunological studies. Ann Neurol. 1977;1:72-8.

5 O'Neill JH, Murray NMF, Newsom-Davies J. The Lambert-Eaton myasthenic syndrome: a review of 50 cases. Brain 1988;111:577-96.

6 Graus F, Elkon KB, Cordon-Cardo C, Posner J. Sensory neuronopathy and small cell lung cancer: anti-neuronal antibody that also reacts with the tumour. $\mathrm{Am} \mathrm{J} \mathrm{Med}$ 1986;80:45-52.

7 Babikian VL, Stefansson K, Dieperink ME, Arnason BGW, Marton L, Levy BE. Paraneoplastic myelopathy: antibodies against protein in normal spinalcord and antibodies against protein in normal spinal

8 Greenlee JE, Lipton HL. Anticerebellar antibodies in serum and CSF of a patient with oat cell carcinoma of lung and paraneoplastic cerebellar degeneration. Ann Neurol 1986; 19:82-85.

9 Ridley A, Kennard C, Scholtz CL, Buttner-Ennever JA, Summers B, Turnbull A. Omnipause neurones in two cases of opsoclonus associated with oat cell carcinoma of the lung. Brain 1987;110:1699-709.

10 Kornguth SE, Kalinke T, Scjutta H, Dahl D. Antineurofilament antibodies in the sera of patients with small cell carcinoma of the lung and with visual paraneoplastic syndrome. Cancer Research 1986;46:2588-95.

11 Vincent A, Lang B, Newsom-Davis J. Autoimmunity to the voltage-gated calcium channel underlies the Lambertvoltage-gated calcium channel underlies the LambertEaton myastheic syndrome, a paraneop

12 Newsom-Davis J, Murray NMF. Plasma exchange and immunosupressant drug treatment in the Lambert-Eaton myasthenic syndrome. Neurology 1984;34:480-5.

13 Ewing DJ. Recent advances in the non-invasive investiga tion of diabetic autonomic neuropathy. In: Bannister $\mathbf{R}$ ed. Autonomic failure. Oxford: University Press, 1988: 667-89.

14 Hawley RJ, Cohen MH, Saini N, Armbrustmacher VW. The carcinomatous neuromyopathy of oat cell lung cancer. Ann Neurol. 1980;7:65-72.

15 Park DM, Johnson RH, Crean GP, Robinson JF. Orthostatic hypertension in bronchial carcinoma. $\operatorname{Br} M J$ 1972;3:510-1.

16 Maier HC, Sommers SC. Neuroendocrine carcinoma of lung associated with bradycardia and episodic cardiac asystole. Ann Thoracic Surgery 1986;41:560-2.

17 Strandell T. Circulatory studies on healthy old men. $A c t$ Med Scand. 1964;(Suppl) 414:1-44.

18 Lambert EH, Rooke ED, Eaton LM, Hodgson CH. Myasthetic syndrome occasionally associated with bronchial neoplasm: neurophysiologic studies. In: Viets HR ed. Myasthenia gravis. Springfield: Thomas, 1961:362-410.

19 Yesner R, Gelfman NA, Feinstein AR. A reappraisal of histopathology in lung cancer and correlation of cell types with antecedent cigarette smoking. Am Rev Resp Dis 1973;107:790-7.

20 Croft PB, Wilkinson $M$. The incidence of carcinomatous neuromyopathy with special reference to lung and breast carcinoma. In: Brain R and Norris FH, eds. The remote effects of cancer on the nervous system. New York: Grune and Stratton, 1965:44-54.

21 Gomm SA. Neuromuscular disorders and lung cancer (Thesis). Manchester: University of Manchester. 1986

22 De la Monte S, Hutchins GM, Moore GW. Paraneoplastic syndromes and constitutional symptoms in prediction of
metastatic behaviour of small cell carcinoma of the lung. metastatic behaviour of smal

23 Office of Population Censuses and Surveys. 1986 mortality statistics: cause (England and Wales). London: OPCS 1988:104-5.

24 Brain R, Henson RA. Neurological syndromes associated with carcinoma. Lancet 1958;ii:971-5.

25 Shy GM, Silverstein I. A study of the effects upon the motor unit by remote malignancy. Brain 1965;88:515-28.

26 Rapoport SI. Blood-brain barrier in physiology and medicine. New York: Raven Press, 1976:74-9, 140-2. 